\title{
Avaliação do grau de independência de idosos residentes em instituições de longa permanência*
}

\author{
ASSESSMENT OF THE LEVEL OF INDEPENDENCE OF ELDERLY RESIDENTS \\ IN LONG-TERM CARE INSTITUTIONS
}

\author{
EVALUACIÓN DE EL GRADO DE INDEPENDENCIA DE RESIDENTES \\ EN HOGARES PARA ANCIANOS
}

Maria Odete Pereira Hidaldo de Araújo', Maria Filomena Ceolim²

* Extraído da disser-
tação "O autocuidado
em idosos indepen-
dentes residentes em
instituições de longa
permanência”, Pro-
grama de Pós-gra-
duação de Enferma-
gem, Faculdade de
Ciências Médicas,
Universidade Esta-
dual de Campinas
(FCM-UNICAMP),
2003.Campinas, SP,
Brasil.
1 Mestre em Enferma-
gem pela UNICAMP,
Professor Assistente
III, Faculdades Inte-
gradas Teresa D'Ávila
- Lorena, SP, Brasil
m.odetepereira@
uol.com.br
2 Professor Doutor,
Departamento de
Enfermagem, FCM-
UNICAMP.
Campinas, SP, Brasil.
fceolim@
fcm.unicamp.br

\section{RESUMO}

As instituições de longa permanência abrigam um grande número de idosos dependentes, embora essa não seja condição sine qua non para que ocorra a institucionalização do idoso. O objetivo deste estudo foi identificar o grau de independência para a realização de atividades da vida diária (AVDs) dos idosos residentes nas instituições da cidade de Taubaté-SP, segundo avaliação baseada no Índice de Independência nas AVDs de Katz, realizada em dezembro de 2001 e repetida em maio de 2002. A primeira avaliação, feita com todos os residentes com idade igual ou superior a 60 anos (139 mulheres e 48 homens), mostrou que 70 idosos eram considerados independentes para o desempenho das AVDs, sendo que, dentre eles, 52 idosos tinham entre 70 e 89 anos. A segunda avaliação mostrou que 53 idosos mantiveram-se independentes. Os dados corroboram estudos anteriores que apontam para o declínio da capacidade funcional de idosos institucionalizados.

\section{DESCRITORES}

Saúde do idoso.

Instituição de longa

permanência para idosos.

Atividades cotidianas.

Avaliação geriátrica.

\section{ABSTRACT}

Long-term care institutions shelter a large number of dependent elderly, although dependence is not the sine qua non condition for elderly institutionalization. This study aimed at identifying the independence level for the performance of activities of daily living (ADLs) of elderly residents in long-term care institutions in the city of Taubaté, State of São Paulo, according to an evaluation based on Katz's Index of Independence in ADLs. The first assessment was conducted in December of 2001 with all residents aged 60 or more (139 females and 48 males). Seventy subjects were considered independent for ADLs, 52 of them aged between 70 and 89 . The second assessment, performed in May of 2002, revealed that 53 subjects remained independent for ADLs. The results corroborate previous studies that point to a decline in the independence level of institutionalized elderly subjects.

\section{KEY WORDS}

Health of the elderly.

Homes for the aged.

Activities of daily living.

Geriatric assessment.

\section{RESUMEN}

Las instituciones asilares abrigan un gran número de ancianos dependientes, aunque esta no sea la condición sine qua non para que ocurra la institucionalización del anciano. Este estudio investigó el grado de independencia para la ejecución de actividades de la vida diaria (AVDs) de los ancianos residentes en las instituciones asilares de la ciudad de Taubaté, Brasil, según la evaluación basada en el Índice de Independencia en AVDs de Katz, efectuada en diciembre de 2001 y en mayo de 2002. En la primera evaluación, hecha con todos los individuos con 60 años o más (139 mujeres y 48 hombres), 70 ancianos fueron considerados independientes según el Índice de Katz mientras, 52 de estos ancianos tenían entre 70 y 89 años. La segunda evaluación mostró que 53 ancianos mantuvieron su nivel de independencia. Los hallazgos confirmaron lo estudios anteriores que apuntan para la declinación de la capacidad funcional de ancianos institucionalizados.

\section{DESCRIPTORES}

Salud del anciano.

Hogares para ancianos.

Actividades cotidianas.

Evaluación geriátrica. 


\section{INTRODUÇÃO}

Atualmente observa-se, no mundo todo, o aumento absoluto e proporcional da população idosa e, sabendo-se que o declínio da capacidade funcional aumenta com a idade, todos os esforços devem ser envidados no sentido de prevenir a dependência física e de retardá-la o máximo possível, para que o idoso possa viver por mais tempo no seu ambiente familiar ${ }^{(1)}$.

Voltados para percepções sobre independência, autores focaram predominantemente sobre as habilidades funcionais que dispensam ajuda. Não é surpreendente que o foco esteja restrito às medidas usadas por médicos para avaliar a independência entre pessoas idosas. Vários programas descrevem métodos, ou seja, medidas para mensurar a capacidade de pessoas idosas com relação às suas habilidades para realizar as atividades de vida diária. Outros autores descrevem o uso de medidas psicométricas para avaliar níveis de funcionamento e deterioração cognitiva ao longo do tempo ${ }^{(2)}$.

A avaliação da capacidade funcional é relevante em Gerontologia, como indicativo de qualidade de vida do idoso. O desempenho das atividades de vida diária é considerado um parâmetro aceito e legítimo para firmar essa avaliação, sendo utilizado pelos profissionais da área de saúde, e de extrema valia para o enfermeiro, para avaliar graus de dependência de seus clientes. Pode-se entender avaliação funcional, dentro de uma função específica, como sendo a avaliação da capacidade de autocuidado e de atendimento às necessidades básicas diárias, ou seja, do desempenho das atividades de vida diária ${ }^{(3-4)}$.

Sabe-se que à medida que o ser humano envelhece, muitas tarefas do cotidiano, consideradas banais e, portanto, de fácil execução, vão paulatinamente e muitas vezes de forma imperceptível, tornando-se cada vez mais difíceis de serem realizadas, até que o indivíduo percebe que já depende de outra pessoa para tomar um banho, por exemplo.

A transferência de um idoso de sua casa para a instituição tem um potencial para produzir danos como: depressão, confusão, perda do contato com a realidade, despersonalização e um senso de isolamento e separação da sociedade ${ }^{(5)}$.

Há muitas escalas que avaliam a capacidade funcional. O Índice de Katz, devido à praticidade de sua aplicação e sua confiabilidade, demonstradas em estudos semelhantes, mostrou-se adequado para o propósito das pesquisadoras ${ }^{(6-7)}$. A utilização do Índice de Katz demonstrou que esse instrumento beneficia a avaliação de indivíduos idosos, de doentes crônicos e daqueles em longos períodos de recuperação hospitalar ou, ainda, podia ser utilizado para avaliar a capacidade funcional do indivíduo na comunidade ${ }^{(6)}$. As ativida- des contempladas são descritas como Atividades de Vida Diárias (AVDs), pois se relacionam ao cotidiano do ser humano.

Trata-se de uma escala que permite atribuir diferentes graus de independência funcional aos sujeitos nos atos de: banhar-se, vestir-se, usar o banheiro para eliminações, mobilizar-se da cama para a cadeira, ter continência das eliminações e alimentar-se. A independência significa que a função é realizada sem supervisão, direção ou ajuda, sendo essa avaliação baseada na situação real e não na capacidade do sujeito. Quando um indivíduo se nega a cumprir uma função, considera-se que não a realiza, embora possa ter capacidade para fazê-la. Os graus considerados para a independência ou dependência funcional são progressivos, desde a independência total para todas as funções (grau A), até a dependência total para realizar as seis funções avaliadas (grau G).

Nas instituições asilares de longa permanência a dependência física é muitas vezes estimulada, pois os próprios funcionários preferem ajudar os idosos nas suas atividades, quando esses já apresentam inabilidade para executar tarefas simples, embora não sejam incapazes para fazê-las ${ }^{(5)}$.

\section{OBJETIVO}

Considerando-se que a institucionalização pode resultar em declínio funcional, com conseqüente perda da independência para desempenho das AVDs, este estudo teve como objetivo avaliar o grau de independência para a realização de AVDs dos idosos residentes nas instituições asilares da cidade de Taubaté - SP, por meio do Índice de Katz; e avaliar novamente, após cinco meses, os idosos classificados inicialmente como independentes (grau A de Katz), visando identificar possíveis mudanças.

\section{MÉTODO}

Este estudo é do tipo exploratório e descritivo, com abordagem quantitativa.

A pesquisa foi realizada em três instituições de longa permanência definidas como abrigo para idosos localizadas em Taubaté. Neste estudo, as instituições foram identificadas por meio de algarismos romanos (Instituição I, II e III).

Participaram do estudo todos os residentes com idade igual ou superior a 60 anos completos (critério de inclusão no estudo), observando-se a definição de idoso da Organização Mundial de Saúde para os países em desenvolvimento, como o Brasil ${ }^{(8)}$. Foram observados os seguintes critérios de exclusão dos sujeitos: idade inferior a 60 anos completos na ocasião da coleta de dados; não estar cadastrado como residente em uma das instituições estudadas. 
O instrumento utilizado no estudo foi o Índice de Katz (Anexo).

A coleta de dados foi realizada em duas etapas: em dezembro de 2001 e maio de 2002. Na primeira etapa, foram observados todos os idosos que atendiam aos critérios de inclusão e, na segunda etapa, todos os sujeitos que haviam sido classificados anteriormente com o grau A de Katz. Os próprios sujeitos, se capazes de responder com coerência, participavam das observações respondendo a algumas questões do Índice de Katz. As respostas e as observações foram conferidas com os auxiliares de enfermagem responsáveis pelo atendimento aos residentes.

Os dados foram tabulados e processados em banco de dados eletrônico no programa Microsoft ${ }^{\circledR}$ Excel 97 (Sistema Operacional Windows98, Microsoft Corporation, Inc.), sendo obtidas tabelas de freqüência para a classificação dos idosos em diferentes graus de dependência, faixa etária e sexo.

A pesquisa foi aprovada pelo Comitê de Ética em Pesquisa da Faculdade de Ciências Médicas da UNICAMP (Parecer CEP 294/2001, de 13 de novembro de 2001). Na elaboração do Termo de Consentimento Livre e Esclarecido e nas garantias dos direitos dos sujeitos observados, foram aten- didas as recomendações da Resolução 196/96 do Conselho Nacional de Saúde para pesquisas com seres humanos. O Termo foi assinado pelo próprio idoso ou, se necessário, por representante legal da instituição.

\section{RESULTADOS}

A observação para avaliação do grau de independência para desempenho de AVD foi realizada em 187 indivíduos com 60 anos de idade ou mais. O número de sujeitos estudados em cada instituição é apresentado no Gráfico 1.

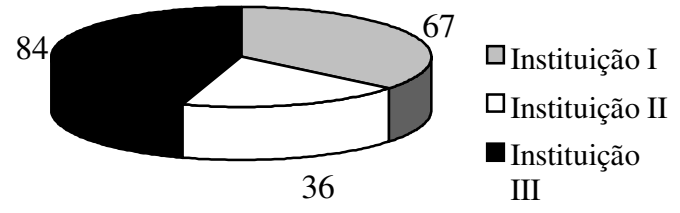

Gráfico 1 - Número de sujeitos estudados em cada instituição - Taubaté - 2001

Os resultados obtidos com o Índice de Katz na primeira etapa do estudo (dezembro de 2001) encontram-se na Tabela 1, a seguir.

Tabela 1 - Distribuição dos sujeitos segundo sexo, faixa etária e Índice de Katz na primeira etapa - Taubaté - 2001

\begin{tabular}{|c|c|c|c|c|c|c|c|c|c|c|c|c|c|}
\hline \multirow{3}{*}{$\begin{array}{l}\text { Índice } \\
\text { de } \\
\text { Katz }\end{array}$} & \multicolumn{10}{|c|}{ Faixa etária (em anos) } & \multirow{2}{*}{\multicolumn{3}{|c|}{ Total }} \\
\hline & \multicolumn{2}{|c|}{$60-69$} & \multicolumn{2}{|c|}{$70-79$} & \multicolumn{2}{|c|}{$80-89$} & \multicolumn{2}{|c|}{$90-99$} & \multicolumn{2}{|c|}{100 ou mais } & & & \\
\hline & M & $\mathrm{F}$ & M & $\mathrm{F}$ & M & $\mathrm{F}$ & M & $\mathrm{F}$ & M & $\mathrm{F}$ & M & $\mathrm{F}$ & Geral \\
\hline $\mathrm{A}$ & 4 & 10 & 4 & 22 & 3 & 23 & - & 4 & - & - & 11 & 59 & 70 \\
\hline B & - & 1 & 1 & 1 & - & 2 & - & - & - & - & 1 & 4 & 5 \\
\hline $\mathrm{C}$ & 6 & 5 & 4 & 2 & 2 & 8 & 1 & - & - & 1 & 13 & 16 & 29 \\
\hline $\mathrm{D}$ & - & 1 & 5 & 1 & - & 1 & - & - & - & 1 & 5 & 4 & 9 \\
\hline $\mathrm{E}$ & 2 & - & - & 2 & - & 1 & - & - & - & - & 2 & 3 & 5 \\
\hline $\mathrm{F}$ & - & 3 & 1 & 4 & - & 2 & - & - & - & - & 1 & 9 & 10 \\
\hline $\mathrm{G}$ & 5 & 8 & 6 & 17 & 3 & 12 & 1 & 4 & - & 1 & 15 & 42 & 57 \\
\hline Outro & - & - & - & 1 & - & 1 & - & - & - & - & - & 2 & 2 \\
\hline Total & 17 & 28 & 21 & 50 & 8 & 50 & 2 & 8 & 0 & 3 & 48 & 139 & 187 \\
\hline
\end{tabular}

Observou-se, como mostra a Tabela 1, o predomínio dos sujeitos do sexo feminino, correspondendo a $74 \%$ do total de residentes (139). Quanto à distribuição em faixas etárias, a maioria dos sujeitos tinha idade entre 70 e 79 anos (38\%, ou 71 ), seguindo-se a faixa de 80 a 89 anos $(31 \%$, ou 58$)$ e de 60 a 69 anos (24\%, ou 45).

Verificou-se que, no conjunto das três instituições, 70 sujeitos podiam ser considerados independentes para o desempenho das AVDs segundo o Índice de Katz ${ }^{(2)}$, correspondendo a $37 \%$ do total de idosos residentes.
Destaca-se que, dentre os 70 sujeitos independentes, 59 eram mulheres, correspondendo a $42 \%$ de um total de 139 mulheres residentes, e 11 pertenciam ao sexo masculino, representando $23 \%$ do total de 48 homens residentes. Verificou-se ainda que 26 sujeitos, ou seja, $37 \%$ dentre os 70 sujeitos independentes, encontravam-se na faixa etária de 80 a 89 anos de idade.

$\mathrm{Na}$ Instituição I, 47 sujeitos eram independentes, correspondendo a $70 \%$ dos 67 residentes no local; na Instituição II, dez dentre os 36 residentes eram independentes, 
ou seja, 28\%; e, na Instituição III, apenas 13 sujeitos foram classificados como totalmente independentes, representando $15 \%$ dentre 84 residentes .
Os 70 idosos independentes (grau A de Katz) foram submetidos à segunda observação de acordo com o Índice de Katz, cinco meses após a primeira. Os resultados encontram-se na Tabela 2.

Tabela 2 - Distribuição dos sujeitos classificados no grau A de Katz na primeira etapa do estudo segundo sexo, faixa etária e Índice de Katz na segunda etapa - Taubaté - 2002

\begin{tabular}{|c|c|c|c|c|c|c|c|c|c|c|c|}
\hline \multirow{3}{*}{$\begin{array}{l}\text { Índice de } \\
\text { Katz }\end{array}$} & \multicolumn{8}{|c|}{ Faixa etária (em anos) } & \multirow{2}{*}{\multicolumn{3}{|c|}{ Total }} \\
\hline & \multicolumn{2}{|c|}{$60-69$} & \multicolumn{2}{|c|}{$70-79$} & \multicolumn{2}{|c|}{$80-89$} & \multicolumn{2}{|c|}{$90-99$} & & & \\
\hline & $\mathrm{M}$ & $\mathrm{F}$ & M & $\mathrm{F}$ & $\mathrm{M}$ & $\mathrm{F}$ & M & $\mathrm{F}$ & M & $\mathrm{F}$ & Geral \\
\hline $\mathrm{A}$ & 3 & 8 & 4 & 20 & 2 & 17 & 0 & 3 & 9 & 48 & 57 \\
\hline $\mathrm{B}$ & 0 & 0 & 0 & 0 & 0 & 1 & 0 & 0 & 0 & 1 & 1 \\
\hline $\mathrm{C}$ & 0 & 1 & 0 & 0 & 0 & 2 & 0 & 0 & 0 & 3 & 3 \\
\hline $\mathrm{D}$ & 0 & 0 & 0 & 0 & 0 & 0 & 0 & 0 & 0 & 0 & 0 \\
\hline $\mathrm{E}$ & 0 & 0 & 0 & 0 & 0 & 0 & 0 & 0 & 0 & 0 & 0 \\
\hline $\mathrm{F}$ & 0 & 0 & 0 & 0 & 0 & 1 & 0 & 0 & 0 & 1 & 1 \\
\hline G & 0 & 0 & 0 & 0 & 0 & 1 & 0 & 0 & 0 & 1 & 1 \\
\hline Outro & 0 & 0 & 0 & 0 & 0 & 0 & 0 & 0 & 0 & 0 & 0 \\
\hline Subtotal & 3 & 9 & 4 & 20 & 2 & 22 & 0 & 3 & 9 & 54 & 63 \\
\hline Demência & 0 & 0 & 0 & 1 & 0 & 1 & 0 & 1 & 0 & 3 & 3 \\
\hline Óbito & 1 & 1 & 0 & 1 & 1 & 0 & 0 & 0 & 2 & 2 & 4 \\
\hline Total & 4 & 10 & 4 & 22 & 3 & 23 & 0 & 4 & 11 & 59 & 70 \\
\hline
\end{tabular}

Verificou-se que seis sujeitos, todos do sexo feminino, apresentaram declínio funcional na segunda etapa do estudo, correspondendo a $9 \%$ dos 70 sujeitos anteriormente classificados no grau A. Outros três sujeitos, também do sexo feminino, apresentaram declínio funcional e cognitivo, representando $4 \%$ do total de sujeitos que pertenciam ao grau A na primeira avaliação. Dentre esses nove sujeitos com declínio funcional ou cognitivo e funcional, seis pertenciam à faixa etária de 80 a 89 anos e uma à faixa etária de 90 a 99 anos. Verificou-se, portanto, que o declínio funcional ocorreu predominantemente entre os idosos com 80 anos e mais, que representaram 54\% (7) dos 13 idosos, anteriormente considerados independentes, que apresentaram declínio funcional ou que faleceram antes da segunda avaliação. Portanto, na segunda avaliação, 57 idosos permaneceram no grau A de Katz, ou seja, independentes para as AVDs, representando $81 \%$ do total de 70 sujeitos classificados nesse grau na primeira etapa.

\section{DISCUSSÃO}

Neste estudo, verificou-se que, dentre 187 idosos institucionalizados, 70 eram considerados independentes para o desempenho das atividades de vida diária, correspondendo a $37 \%$ dos idosos avaliados. Estes dados corroboram os achados de um estudo realizado em 1990, no qual foram encontrados $38 \%$ de idosos institucionalizados independentes para as AVDs ${ }^{(9)}$. Estes resultados contribuem para manter a afirmativa de que a institucionalização ainda está, na maioria das vezes, associada à dependência física e cognitiva ${ }^{(4)}$. Portanto, ao considerar que mais de um terço dos idosos institucionalizados eram independentes para as AVDs, os mesmos precisam ser estimulados para manter-se nesta condição ${ }^{(5,9)}$.

Outras pesquisas recentes, realizadas com o objetivo de avaliar a capacidade funcional e cognitiva de idosos institucionalizados, obtiveram como resultado uma proporção discretamente superior de idosos independentes quando comparadas a este estudo, mas realizaram a avaliação do grau de independência dos idosos em uma única ocasião. Em Belo Horizonte, 84 idosos de uma instituição de longa permanência foram avaliados com o Índice de Katz, sendo observado que $50 \%$ dos idosos eram independentes (grau A), 36,9\% encontravam-se em graus de dependência parcial (B,C,D,E,F) e 13,1\% com dependência total (grau G) ${ }^{(10)}$. Outro estudo avaliou 150 idosos institucionalizados, constatando que $43 \%$ eram independentes; $24 \%$ semi-dependentes e $29 \%$ dependentes totais ${ }^{(11)}$.

Um achado surpreendente deste estudo foi a proporção de idosos independentes que se encontrava na faixa etária de 80 a 89 anos de idade, que representavam 37\% (26) dos 70 sujeitos independentes. Considerando-se a faixa etária elevada, pode-se sugerir que os idosos brasileiros, uma vez 
considerados dependentes, são institucionalizados com menor idade, pois não há quem possa cuidar deles no domicílio $^{(12)}$. Autores apontam que, muitas vezes, o ancião fragilizado e dependente convive no seu lar somente com outro idoso, o qual enfrenta inúmeras dificuldades para o cuidado, que incluem desde o ambiente domiciliar inadequado e a falta de conhecimentos para exercer o papel de cuidador, até a dificuldade de transporte do idoso dependente aos serviços de saúde, quando necessário ${ }^{(13)}$. Por outro lado, os sujeitos de idade mais avançada, embora independentes para o desempenho de AVDs, poderiam ser institucionalizados por outros motivos, tais como problemas financeiros, solidão e dificuldade para o desempenho de atividades instrumentais da_vida diária ${ }^{(5,12-13)}$.

Dentre as instituições estudadas, duas adotavam como critério receber apenas idosos capazes de desempenhar sozinhos todas as AVDs, enquanto a terceira instituição era a única que admitia receber idosos dependentes. Entretanto, os sujeitos totalmente independentes predominavam em apenas uma destas instituições, na qual representavam $70 \%$ do total de residentes, enquanto as outras abrigavam grande proporção de idosos dependentes, que correspondiam a mais de $70 \%$ do total de residentes em cada qual. Deve-se considerar que podem ocorrer situações nas quais os cuidadores estimulem a dependência dos idosos, como durante o banho e a alimentação, tendendo a realizar as ações que os próprios residentes poderiam desempenhar, mesmo que mais lentamente ${ }^{(5)}$. O fato é que mesmo o idoso independente, quando institucionalizado, pode desenvolver diferentes graus de dependência devido à sua dificuldade em aceitar e adaptar-se às novas condições de vida e à falta de motivação e de encorajamento que são comuns no ambiente $\operatorname{asilar}^{(5)}$.

A dependência comportamental é a forma de dependência mais temida pelos idosos. Pode se desenvolver como resultado do desamparo aprendido, num ambiente não responsivo, negligenciador e não contingente, que faz com que o idoso se sinta desamparado e passe a desenvolver um comportamento dependente. Do contrário, o ambiente contingente, pouco exigente e caracterizado por superproteção, também resulta em dependência comportamental como instrumento de controle passivo ${ }^{(14)}$.

Neste estudo, $19 \%$ dos idosos considerados independentes na primeira avaliação apresentaram declínio funcional de graus variados após cinco meses, o que pode ser considerado um percentual expressivo. Deve-se ressaltar que a maioria dos sujeitos observados tinham pelo menos um ano de residência na instituição. Outros autores observaram que os idosos independentes admitidos numa instituição mantiveram-se nesta condição quando avaliados seis meses após, enquanto cerca de $25 \%$ dos idosos que se apresentaram semi-dependentes no momento da internação evoluíram para dependência total, aumentando o número de dependentes totais ( $\mathrm{G}$ de Katz) em 2,5 vezes ${ }^{(15)}$.

O declínio funcional observado neste estudo pode ser explicado em parte por outros autores que concluíram que os cuidadores na instituição de longa permanência parecem não estimular as capacidades dos idosos ainda capazes de desenvolver as atividades básicas e instrumentais da vida diária ${ }^{(15-16)}$. O estímulo à autonomia e independência do idoso institucionalizado é condição sine qua non para a manutenção da sua independência física e comportamental.

Os resultados apontam ainda para a necessidade de encorajamento dos idosos no ambiente institucional, lançando mão de planos individualizados para estimular o seu potencial de autocuidado, a fim de que permaneçam independentes o máximo de tempo possível.

\section{CONCLUSÕES}

Segundo os resultados obtidos, os idosos com grau de independência total para o desempenho das AVDs constituíam 37\% dos residentes nas instituições de longa permanência da cidade de Taubaté, em dezembro de 2001. Uma nova avaliação, cinco meses após, mostrou que 19\% destes sujeitos haviam apresentado declínio funcional, cognitivo e funcional, ou falecido.

\section{CONSIDERAÇÕES FINAIS}

Estudos sobre a condição de independência na velhice versam mais sobre dependência e medidas de avaliação de dependência, do que sobre a independência.

Parece que a dependência do idoso é vista como algo natural e esperado mas, na verdade, sabe-se que quando ele é acometido por patologias que o levam à condição de dependência parcial ou total, é possível ainda reabilitá-lo para que recupere a capacidade de realizar uma ou outra atividade de vida diária. A reabilitação de algumas funções, embora muitas vezes possa parecer insignificante para a família, devolve ao idoso a capacidade do fazer por ele mesmo, ou seja, do autocuidado. 


\section{REFERÊNCIAS}

1. Paschoal SMP. Autonomia e independência. In: Papaléo Neto M, organizador. Gerontologia. São Paulo: Atheneu; 1999. p. $313-26$.

2. Secker J, Hill R, Villeneau L, Parkman S. Concept forum promoting independence: but promoting what and how? Ageing Soc. 2003;23(3):375-91.

3. Diogo MJD'E. Satisfação com a vida e a capacidade funcional em idosos com amputação de membros inferiores [tese livredocência]. Campinas: Universidade Estadual de Campinas; 2001.

4. Diogo MJD'E. Avaliação funcional de idosos com amputação de membros inferiores. Rev Lat Am Enferm. 2003;11(1):59-65.

5. Pavarini SCI. Dependência comportamental na velhice: uma análise do cuidado prestado ao idoso institucionalizado [tese]. Campinas: Universidade Estadual de Campinas; 1996.

6. Katz S, Ford AB, Moskowitz RW, Jackson BA, Jaffe MW Studies of illness in the aged. The index of ADL: a standardized measure of biological and psychosocial function. JAMA. 1963;185(12):914-19.

7. Spector WD. Funcional disability scales. In: Spilker B, editor. Quality of life assessments in clinical trials. New York: Raven Press; 1990. p. 115-29.

8. Organización Mundial De La Salud (OMS). Aplicacion de la epidemiologia al estúdio de los ancianos: informe de um grupo científico de la OMS. Genebra; 1984. (Série Informes Técnicos, n. 706).

9. Barbosa MLJ. A situação dos idosos no Vale do Paraíba: análise nas cidades de Taubaté e Guaratinguetá [tese]. São Paulo: Programa Interunidades, Escola de Enfermagem, Escola de Enfermagem de Ribeirão Preto, Universidade de São Paulo; 1990.
10. Thorun IMR, Marino MA, Santos AGR, Moraes EN. Prevalência de dependência funcional, déficit cognitivo e distúrbios psíquicos em idosos de uma instituição asilar. In: Anais do $3^{\circ}$ Congresso Sul-Brasileiro de Geriatria e Gerontologia; 2001 set. 6-9; Florianópolis. Florianópolis: SBGG-Seção SC; 2001. p. 64 .

11. Moraes ZV, Trigo RR, Palomaro NP, Brito DA, Vainzoff R, Martins AM, et al. Perfil de idosos de uma instituição asilar no município de São Paulo. In: Anais do $13^{\circ}$ Congresso Brasileiro de Geriatria e Gerontologia; 2002 jun. 19-22; Rio de Janeiro. Rio de Janeiro: SBGG-Seção RJ; 2002. p. 449.

12. Kawasaki K, Diogo MJD’E. Assistência domiciliária ao idoso: perfil do cuidador formal - Parte I. Rev Esc Enferm USP. 2001;35(3):257-64.

13. Diogo MJD'E, Ceolim MF, Cintra FA. Orientação para idosas que cuidam de idosos no domicílio. Rev Esc Enferm USP. 2005;39(1):97-102.

14. Baltes MM, Silverberg S. A dinâmica dependência-autonomia no curso da vida. In: Néri AL, organizadora. Psicologia do envelhecimento: temas selecionados numa pespectiva de curso de vida. Campinas: Papirus; 1995. p. 73-110.

15. Guia CM, Lemos RM, Rodrigues HC, Pessolani MA, Camargos E, Toledo MAV, et al. Declínio funcional e cognitivo em idosos após 6 meses de internação em instituição gerontológica. In: Anais do $13^{\circ}$ Congresso Brasileiro de Geriatria e Gerontologia; 2002 jun. 19-22; Rio de Janeiro. Rio de Janeiro: SBGG-Seção RJ; 2002. p. 348.

16.Camargo WCG, Leão MABG. O papel do cuidador na estimulação da autonomia e independência do idoso institucionalizado. In: Anais do $13^{\circ}$ Congresso Brasileiro de Geriatria e Gerontologia; 2002 jun. 19-22; Rio de Janeiro. Rio de Janeiro: SBGG-Seção RJ; 2002. p. 352.

\section{Agradecimentos}

À Coordenação de Aperfeiçoamento de Pessoal de Nível Superior (CAPES) pelo apoio financeiro. 


\section{Anexo \\ Índice de Katz}

\section{Atividades da Vida Diária (AVDs)}

Banhar-se (de esponja, na banheira ou no chuveiro):

Toma banho completamente sem assistência.

Recebe assistência para lavar apenas uma parte do corpo, como: pernas ou costas.

Recebe assistência para lavar mais que uma parte do corpo.

Vestir-se (retirar roupas do armário e do cabide, inclusive roupas de baixo e sobretudo; lidar com fechos e cintos e calçar sapatos):

Apanha as roupas e veste-se completamente sem assistência.

Apanha as roupas e veste-se sem assistência, exceto no amarrar os cordões dos sapatos.

Recebe assistência para arrumar as roupas e vestir-se ou permanece parcial ou completamente sem roupa.

Usar sanitário (ir ao sanitário para as eliminações, limpar-se após as eliminações e arrumar as vestes):

$\square$ Vai ao sanitário, limpa-se e arruma as vestes sem assistência. (pode usar objetos auxiliares como bengala, andador e cadeira de rodas, pode usar comadre/papagaio à noite, esvaziando-os de manhã)

$\square$ Recebe assistência para ir ao sanitário, no limpar-se e arrumar as vestes após as eliminações ou no uso de comadre/ papagaio à noite.

$\square$ Não consegue usar o sanitário para as eliminações.

Deitar e levantar da cama e sentar e levantar da cadeira:

$\square$ Sobe e desce da cama assim como senta-se e levanta-se da cadeira sem assistência (pode estar usando objeto auxiliar com bengala, andador).

$\square$ Sobe e desce da cama assim como senta-se e levanta-se da cadeira com assistência.

Não sai da cama, acamado completamente.

Continência das eliminações:

$\square$ Tem controle completo das eliminações urinária e intestinal.

$\square$ Tem ocasionais "acidentes".

A assistência ajuda a manter o controle da micção (dos que usam cateter ou que são incontinentes).

\section{Alimenta-se:}

Alimenta-se sem assistência.

Alimenta-se por si, exceto para cortar a carne e passar manteiga no pão.

Recebe assistência para alimentar-se.

Recebe alimentação por gavagem ou por via enteral. 


\section{Índice de independência nas atividades de vida diária}

O índice do grau de independência nas atividades da vida diária se baseia numa avaliação da independência ou dependência funcional dos pacientes para banhar-se, vestir-se, usar o sanitário, mobilizar-se, ser continente e comer sem ajuda. As definições de independência e dependência funcionais aparecem na abaixo do índice.

A - Independente para comer, ser continente, mobilizar-se, usar o sanitário, vestir-se e banhar-se.

B - Independente para realizar todas estas funções, exceto uma.

C - Independente para realizar todas as funções, exceto banhar-se e outra função mais.

D - Independente para realizar todas as funções, exceto para banhar-se, vestir-se e outra função mais.

E- Independente para realizar todas as funções, exceto banhar-se, vestir-se, usar o sanitário.

F - Independente para realizar todas as funções, exceto banhar-se, vestir-se, usar o sanitário, mobilizar-se e outra função mais.

G- Dependente para realizar as seis funções.

Outro - Dependente para realizar pelo menos duas funções, mas não pode ser classificado em C, D, E e F.

Independência significa que a função se cumpre sem supervisão, direção ou ajuda pessoal ativa, exceto a que se indica em cada caso. Baseia-se na situação real e não na capacidade. Quando um paciente se nega a cumprir uma função, se considera que não realiza essa função, quando se estima que está capacidade para fazê-lo.

\section{Banho (esponja, ducha ou banheira)}

Independente: necessita de ajuda apenas para lavar uma parte do corpo (como o dorso ou uma extremidade incapacitada) ou lava por si só todo o corpo.

Dependente: necessita de assistência para lavar mais que uma parte do corpo; assistência para entrar e sair da banheira ou não se banha sozinho

\section{Mobilidade}

Independente: deita-se e levanta-se sem auxílio e senta e se levanta da cadeira sem ajuda (usa ou não aparelhos ortopédicos para sustentar-se).

Dependente: mecessita de ajuda para deitar-se e levantar-se da cama ou para sentar-se e levantar-se da cadeira; não pode efetuar um ou mais desses movimentos.

\section{Vestir-se}

Independente: retira as peças de vestir do armário e cabides, inclusive roupas de baixo e sobretudo; veste-se sem auxílio, lida com fechos, cintos e botões e calça sapatos; o ato de amarrar os cordões dos sapatos está excluído.

Dependente: não se veste sozinho ou permanece parcialmente despido.

\section{Continência}

Independente: Tem controle total da micção e da defecação.

Dependente: incontinência parcial ou total da micção ou defecação; controle parcial ou total mediante enemas, cateteres, ou o uso regular de papagaios ou comadres.

\section{Uso do Sanitário}

Independente: Vai para o banheiro sem ajuda; pode sentar-se e levantar-se do vaso; limpa-se e veste-se; pode usar papagaio ou comadre, durante a noite e usa ou não objetos auxiliares (como bengala, andador ou cadeira de rodas) para sustentar-se.

Dependente: usa comadre ou papagaio ou recebe assistência para usar o banheiro

\section{Comer}

Independente: pega a comida do prato ou objeto equivalente e a leva à boca (excluem-se cortar a carne e a preparação prévia de alimentos como passar manteiga no pão)

Dependente: necessita de ajuda para comer; não ingere nenhum alimento ou recebe o alimento por via parenteral. 